\title{
IMPEDANCE CONTROL OF High-PRECiSION GEARED SERVo DRIVES WITH TWO MOTORS FOR TECHNOLOGICAL ROBOTS
}

\author{
Yury Ilyukhin, Ruslan Kolesnichenko \\ Moscow State University of Technology “STANKIN”, Vadkovskiy per. 1, Moscow 127994, Russian Federation
}

\begin{abstract}
The article discusses the possibility and advisability of application of high precision geared servo drives with two motors for milling, drilling and deburring by technological robots. We conducted an analysis of the dynamic properties of drives containing elastic mechanical transmission with backlash. We offered advice on selecting of the structure and parameters of regulators. The results of the theoretical research and computer simulation confirm the high precision of movements and the effectiveness of the proposed algorithms of impedance control, which considerably improves dynamic properties of drives of technological robots.
\end{abstract}

Keywords: technological robots; servo drives; impedance control; high precision; backlash; two motors servo drive
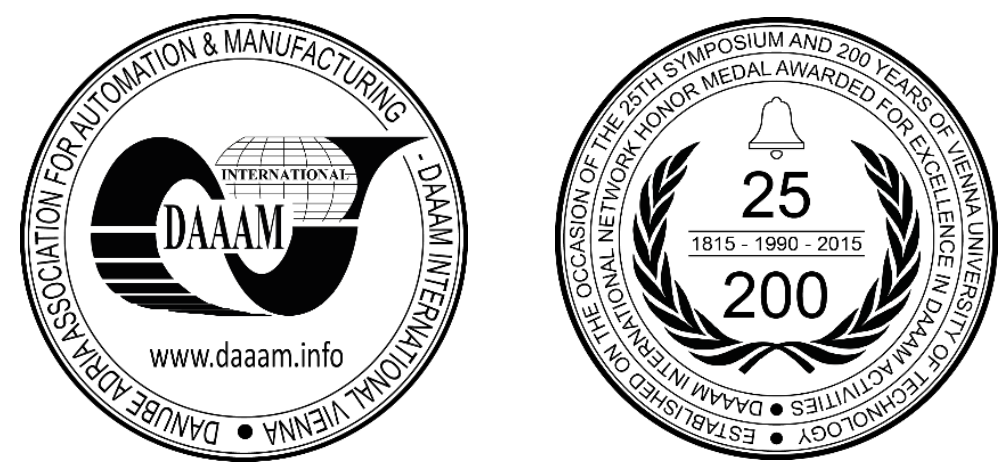

This Publication has to be referred as: Ilyukhin, Y[uri] \& Kolesnichenko, R[uslan] (2016). Impedance Control of HighPrecision Geared Servo Drives with Two Motors for Technological Robots, Proceedings of the 26th DAAAM International Symposium, pp.0599-0607, B. Katalinic (Ed.), Published by DAAAM International, ISBN 978-3-90273407-5, ISSN 1726-9679, Vienna, Austria

DOI: $10.2507 / 26$ th.daaam.proceedings.081 


\section{Introduction}

Modern manufacture is setting high requirements to accuracy of movements of technological robots. It relates to the robots, implementing milling, drilling, deburring, precise laser and plasma cutting, assembly of miniature devices, marking, coordinate measuring of products with large dimensions and complex shape of the surface. To do such operations robots must have significant service areas and they have an open kinematic chain. Admissible deviations of the actual position of the effector (tool) from its desired position should not exceed several tens of micrometres. High precision of movements is especially necessary at analytical programming of technological robots, when the coordinated correction of supporting points under the method of training is not realized. In this case, it is a question about the absolute robot positioning accuracy, not about the positioning repeatability.

Technological robots can be constructed on geared or gearless drive modules. In contrast to the machine tool manufacture, where gearless drives are widespread [1], in the robotics geared servo drives with closed position control loop of the motor shaft are generally applied [2,3]. The usage of gears, as a rule, improves weight and size, energy and value indicators of robots. However, in this case the mechanical transmission turns out to be in an opened loop, beyond the closed position control loop. The accuracy of the effector movements reduces because of an influence of elastic deformations and backlashes, proper to mechanical transmissions. Drive errors are often the prevailing factors, they have an extensive influence on the accuracy of the tool movements and the quality of the performance of technological operation. This fact is confirmed by the results of experimental researches [4]. They show that the largest component of the position error is caused by the backlash effect in the mechanical transmissions of the drives of robot portable degrees of freedom. At the action of the moments of unbalance forces of manipulation mechanism and of the forces from milling, the backlash and the elastic compliance of mechanical transmission may cause a deviation of the effector position from its desired value, up to several $\mathrm{mm}$. Therefore, traditional geared drives are not effective to create precision technological robots.

Significant efforts of scientists are focused on increasing accuracy of servo systems with elastic mechanical transmissions and improving control methods for them [3, 5-8]. The suggestions for the improvement of properties of drives are based on an introduction of various correcting and compensating links, but in practice they often turn out to be inefficient due to the influence of nonlinearity of the type "dead zone". The backlash influence is compensated by adjusting the control impacts, generated by $\mathrm{CNC}$, in the machine-tool manufacture [8-10]. However, this method has not been widely adopted in technological robotics.

The usage of the drives with two coordinately controlled motors is to be more efficient. One of the motors, the primary, is the part of the principal servo drive, closed by a position control loop of the manipulator link, and the other motor forms the part of the torque former, counteracting the primary motor. The features of the construction of such type of drives are considered in $[3,11]$. The robot effector can follow a predetermined path with high accuracy due to a significant increase of stiffness of the geared drive with two motors. However, the usage of the drives with two motors is followed by the appearance of two serious problems. At first, an introduction of the elastic gear with backlash into the control loop may result in arising intensive self-oscillations, which must be eliminated. At second, electromechanical subsystems are inclined to an appearance of relatively low-frequent weakly damped oscillations, which are to be damped.

The imperfection of the mathematical model of manipulator, used for kinematic motion control, also effects the robots accuracy. The deviation of the effector from its desired position can be caused by inaccurate knowledge of the lengths of the links and the misalignment of the axes. In this case in order to increase the robots accuracy calibration is provided $[12,13]$. Along with that, the robot accuracy is often effected to the great extent by the errors of the geared drives.

Examples of application of the drives with two motors aimed to compensate an influence of the backlash of mechanical transmission can be found in $[3,11,14,15]$. There is a structure of the drive with two motors controlled by $\mathrm{CNC}$ and which is created on the master/slave principle in [14]. However, only velocity control of the control object is considered there. Different control strategies of the drives with two motors for manipulators with parallel kinematic structure are analyzed in $[11,15]$. However, the conditions, at which limit cycles are excluded and the required oscillation damping is implemented at the same time are considered insufficiently in the literature. The decision, proposed by the article, is based on impedance control and is oriented to technological robotics. It differs from the known decisions, leading to an increase of the robot compliance [16-19], in the purposeful increase of dynamic stiffness of the drives and in oscillations damping in the servo drive with two motors with elastic gears, possessing backlash.

The present paper has been carried out under the financial assistance of the Ministry of Education and Science of the Russian Federation in the framework of the state assignment in the field of scientific activities (assignment № 2014/105, project № 1678).

\section{The features of the structure of the high-precision geared servo drives with two motors for technological robots}

The precision servo drive of technological robot under consideration contains two actuating motors, mechanically connected with the control object by gears and the output mechanical transmission (Fig. 1). The first of them directly takes part in the position control of the control object. The second motor is a part of a drive of the torque resistance. Transistor power converters create the required tension and winding currents. 


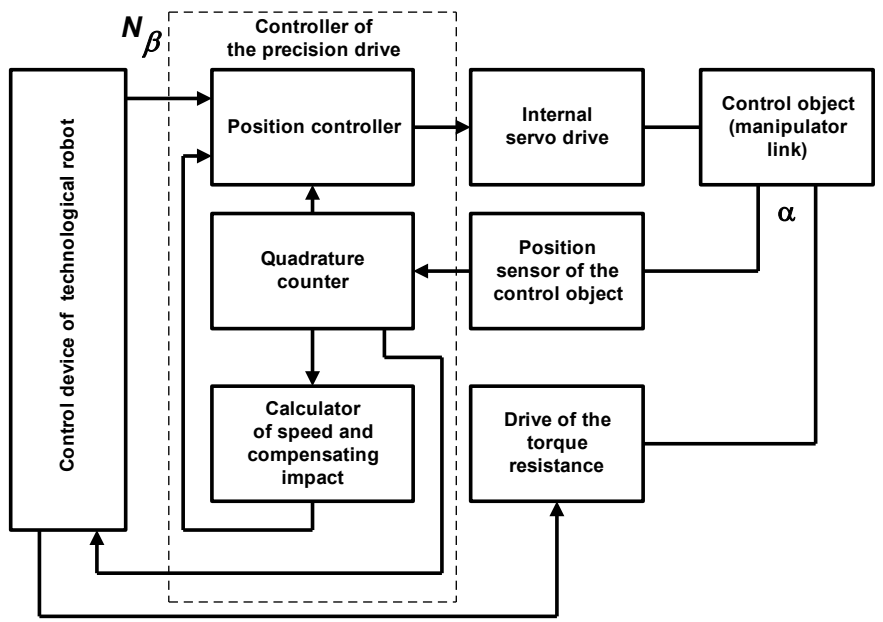

Fig. 1. A structure of the precision servo drive of technological robot

A position sensor is installed on the shaft of the first motor, which allows forming a servo drive, closed by the rotary angle of the motor shaft. This geared servo drive has a traditional structure in the form of 3 subordinated control contours: an external position control contour of the motor shaft, an embedded rotation velocity control contour of the motor shaft and an internal currents control contour of the motor windings. This drive acts as a slave and it is located in the primary position control contour of the manipulator link, belonged to the primary precision drive, and so, it can be called as an internal servo drive.

The drive of the torque resistance carries out the functions of the active controlled backlash-eliminating device. It represents a torque drive, closed by the currents control loop of its' motor windings, or a complete drive, which is closed by the rotation velocity control loop of the motor shaft and which operates in a mode of the torque limiting. This ensures the fact that the torque, developed by the drive of the torque resistance, can be adjusted and constrained at the desired level with high precision. This is one of the features of the precision drive under consideration.

A robot control device implements the control of such precision drive. It generates control impacts to the controller of the precision drive, and that generates control impacts, supplied to the input of the primary drive controller. The last one is not surely to be a detached device. The robot control device can realize its functions.

The accuracy of the precision servo drive considerably depends on the accuracy and the resolution of the position sensor, installed on the shaft controlled by the drive of the manipulator link. Therefore, it is advisable to use a precise incremental encoder as such sensor in conjunction with the quadrature counter. Thus, two position sensors are used in the suggested variant of drive. One of them is installed on the motor shaft, the other - on the manipulator link axis. The total quantity of the control loops in the position control channel of the manipulator link is four.

\section{An idea of the construction of the high-precision geared servo drive for technological robots and a theoretical research of its dynamic properties}

The feature of the servo drive with the suggested structure is that the position control contour of the control object, which is a manipulator link, includes a nonlinear mechanical subsystem. It consists of a gear and a mechanical control object (manipulator link), and at the same time the gear possesses elasticity and backlash. The backlash presence can be reflected in a mathematical model of the drive by using a link with nonlinearity of the type "dead zone", as suggested in [3]. This presence is an evidence of the possibility of the appearance of oscillations in the drive with the described structure. One of the tasks for the creation of the qualitative servo drive, is concluded in a choice of the required moment of forces, achieved by the drive of the torque resistance, and in a choice of the parameter values of the control algorithms, which exclude self-oscillations and which are realized by a controller of the precision drive.

The main idea, underlying a definition of the required torque of the drive of the torque resistance and a synthesis of the controlling means of the precision drive, closed by a position control loop of the control object, is concluded in the following. An implementation of the harmonic linearization method allows us to replace the nonlinear link of the type "dead zone" by its harmonic linearization coefficient $q(A)$. The value of this coefficient influences on the choice of the parameter values of the servo drive controllers. We suggest that the torsional angle of the equivalent elastic element of the gear varies at a harmonic law. A linearized equation of the mechanical transmission can be written as

$$
M_{T r}=C_{E q}\left(1+T_{E} p\right)\left(\alpha_{m} k_{G}^{-1}-q\right)
$$

where $M_{T r}, \alpha_{m}$ and $q$-the first harmonic of the moment variations at the output shaft of the gear with a ratio coefficient $k_{G}$, of the rotary angle variation of the motor shaft and of the rotary angle variation of the manipulator link respectively; 
$C_{E q}$ - an equivalent stiffness coefficient of the gear, reduced to its output shaft; $T_{E}$ - time constant of the elastic gear; $p$ - differential operator. At the same time

$$
C_{E q}=C_{G} q(A)_{M I N} ; T_{E}=\chi_{G} / C_{G},
$$

where $\chi_{G}-$ a loss coefficient on an internal viscous friction in the mechanical transmission; $C_{G}-$ a stiffness coefficient of the mechanical transmission.

Proceeding from the reasons of ensuring stability of the servo drive, the values of the gain coefficients of its controllers should be determined at the minimal value $q(A)_{M I N}$ of the harmonic linearization coefficient $q(A)$, which promotes the minimal value of the $C_{E q}$, as the stability margins in this case are less than at the other values of $q(A)$. At the absence of the moment of external forces $q(A)_{M I N}=0$. Therefore, without the drive of the torque resistance, any structures and control algorithms can not guarantee the lack of self-oscillations in the servo drive of the type under consideration, as it is clarified in [3]. On the other hand, if $q(A)_{M I N}>0$, it is possible to get a drive with no selfoscillations. Consequently, the first task consists in selecting the torque of the drive of the torque resistance so that variations of the coefficient $q(A)$ will be limited. It is also important to select the relevant values of the controller parameters of the drive according to the providing condition of the required stability margins of the harmonically linearized drive model.

The moment of external forces $M_{E x}$, acting the control object, includes the moment, created by the drive of the torque resistance. Because of this moment, oscillations of the motor shaft and the control object shaft happen not about zero values, but in relation to some shift angles, caused by the external moment $M_{E x}$ and the stiffness coefficient $C_{G}$ of the mechanical transmission. The constant component $\theta_{0}$ of the torsional angle $\theta$ of the equivalent elastic element, describing the gear properties, appears in this case. The harmonic linearization of the nonlinear component of the type "dead zone" allows us discovering the variable term on its output and the constant term $\phi$ of the shift angle.

A relation, existing between the minimal value $q(A)_{M I N}$ of the harmonic linearization coefficient $q(A)$, and the values of $M_{E x}, C_{G}$ and a half of the backlash of the mechanical transmission $\sigma$ is the following:

$$
q(A)_{M I N}=f\left\{M_{E x}\left(C_{G} \sigma\right)^{-1}\right\}
$$

while $M_{E x}\left(C_{G} \sigma\right)^{-1}=\phi / \sigma$. Than the $M_{E x}$ is more and the $\sigma$ is less, the range of the possible variations of the harmonic linearization coefficient $q(A)$ is the narrower. Its minimal value $q(A)_{M I N}$ rises with the extension of the $\phi / \sigma$. Its maximum value equals to one and in case the backlash absence too. For example, at $M_{E x}=45 \mathrm{Nm}, C_{G}=250000$ $\mathrm{Nm} / \mathrm{rad}, \sigma=1.4545 * 10^{-4} \mathrm{rad}$ we obtain $q(A)_{\text {MIN }} \approx 0.62$.

We can make a conclusion that the external moment impact results in the fact that the properties of the mechanical transmission with backlash approach the properties of the backlash-free transmission. As a result, the servo drive can be closed by a position control loop of the manipulator link. So, it does not have any static error, caused by elasticity and backlash of the mechanical transmission, and it is able to provide high accuracy of the control object motions. This fact can be interpreted as there is an infinitely large static stiffness of the servo drive. The position errors in this case are caused only by the limited accuracy of the position sensor of the control object.

The dynamic stiffness of the servo drive arises at the action of the varying moment of external forces to the control object, and it also can be increased as a result of the rational choice of the structure and the values of the controllers parameters. Thus, an influence on the impedance of the drive is realized, primarily, by varying the structure of the drive and applying effective control laws.

The mechanical subsystem of the drive consists of the elastic mechanical transmission with backlash and the mechanical control object with a moment of inertia $J_{0}$. Its mathematical model is described by the transfer function

$$
W_{0}(s)=\left(1+T_{E} s\right)\left(\omega_{0}^{-2} s^{2}+2 \xi_{0} \omega_{0}^{-1} s+1\right)^{-1},
$$

where $\omega_{0}=\sqrt{C_{E q} / J_{0}}$ and $\xi_{0}$ - an angular eigenfrequency of oscillations and a coefficient of the relative damping of the mechanical subsystem, while $\xi_{0}=0.5 T_{E} \omega_{0}$. For example, at $C_{E q}=250000 \mathrm{Nm} / \mathrm{rad}$ and $J_{0}=10 \mathrm{kgm}^{2}$, the angular frequency $\omega_{0}=158.1 \mathrm{rad} / \mathrm{s}$. The value of the coefficient $\xi_{0}$ is usually within the range $0.05 \ldots 0.2$.

The internal servo drive and the whole precision drive are digital. At rather high sampling rate of signals over time, which is usually implemented, the properties of such drive are practically the same as the properties of its continuous analog, as shown in [20]. The internal digital servo drive and the mechanical subsystem are dynamically interrelated and 
they form a unified dynamical electromechanical subsystem of the precision drive. Despite the fact, that the internal drive is a servo drive, its motor shaft is shifted under the action of the moment of reaction forces from the elastic gear side. Therefore, the value of the resulting stiffness of the electromechanical subsystem is reduced, and this fact entails the reduction of the resonant frequency of the electromechanical subsystem.

The dynamic properties of the dominant component of the electromechanical subsystem can be characterized in simplified form by the transfer function with an acceptable for practice accuracy. This function represents a relation of function of complex frequency of the rotary angle of the control object $\alpha(s)$ after Laplace transform to a function of complex frequency of the input impact $U_{2}(s)$, supplied to the input of the internal servo drive, after Laplace transform. The function is the following:

$$
W_{E M}(s)=k_{G}^{-1} k_{P S .2}^{-1}\left(1+T_{E} s\right)\left(\omega_{1}^{-2} s^{2}+2 \xi_{1} \omega_{1}^{-1} s+1\right)^{-1}\left(T_{S D .2} s+1\right)^{-1},
$$

where $s$ - the complex number frequency after Laplace transform; $k_{G}$ - gear ratio; $T_{S D .2}-$ time constant of the model of the aperiodic component, reflecting the influence of the internal drive, closed by the position control loop of its shaft, using an encoder with the transfer coefficient $k_{P S .2}, \omega_{1}$ and $\xi_{1}$ - the angular frequency and the damping coefficient, characterising an appearance of the resonant properties of the system. The value of the coefficient $k_{P S .2}$ can be obtained by its linearized characteristic, as shown in [20]. It is worth noticing that $\omega_{1}$ is substantially less than $\omega_{0}$, while the value of the parameter $\xi_{1}$ is small. Therefore, weakly damped oscillations can arise in the electromechanical subsystem. This circumstance considerably makes it difficult to ensure the stability and to expand the bandwidth of the precision drive.

An analysis of eq. (5) shows that in order to implement a position control of the precision servo drive it is possible to apply in its direct control contour an integral controller with the transfer function

$$
W_{I C}(s)=k_{I C} s^{-1}
$$

In order to achieve stability and to obtain acceptable transient processes of the precision servo drive, its cut-off frequency in an opened state is to satisfy the condition

$$
\omega_{C} \leq \omega_{1} \xi_{1}
$$

We consider, that

$$
\omega_{C}=k_{I C} k_{P S} k_{G}^{-1} k_{P S .2}^{-1} \text {. }
$$

where $k_{P S}$ - the sensor transfer coefficient of the primary position feedback of the controlled object, we obtain a requirement to the value of the controller gain coefficient

$$
k_{I C} \leq \omega_{1} \xi_{1} k_{P S}^{-1} k_{G} k_{P S .2} .
$$

The presence of the oscillation component in the electromechanical subsystem demonstrates the necessity of taking measures for damping oscillations in order to improve performance and accuracy of movements of the servo drive.

\section{An improvement of the dynamic properties of geared servo drives with two motors of robots based on the impedance control}

An analysis of the above mathematical model of the electromechanical subsystem has shown that in order to weaken the influence of its resonant properties on the dynamic properties of the precision drive it is advisable to introduce corrective links. They will contribute to an increase of damping of oscillations of this subsystem, which gives us a possibility to increase the cut-off frequency $\omega_{C}$ of the opened loop drive and, as a consequence, to raise the performance and to increase its dynamic stiffness. Researches have shown that a reinforcement of the oscillation damping is achieved by corrective velocity feedback loops of the manipulator links, introduced into the internal servo drives inputs. It can be considered that the parameter $\xi_{1}$ in the transfer function $W_{E M}(s)$ is replaced by the parameter $\xi_{2}$, which desired value is within the range $0.7 \ldots 1$ as a result of such correction.

The value of the coefficient of the velocity feedback of the manipulator link $k_{V F}$ should be determined taking into account the actual value of the angular frequency of the natural oscillations of the electromechanical subsystem $\omega_{1}$ by the formula 
$k_{V F}=k_{P S .2} k_{G} \omega_{1}^{-1}$

We note that frequency $\omega_{1}$ can vary in relation to the configuration, taken by the manipulator in course of technological operations. It is caused by the variation of the moment of inertia $J_{0}$ of the mobile sections of the manipulator, shifted by the drive under consideration. The results of researches show that it is advisable to adjust controllers of the internal drive so that its cut-off frequency in the opened position loop $\omega_{C 2}$ is close to the minimum value $\omega_{0 . M I N}$ of the frequency of natural oscillations of the mechanical subsystem. The frequency $\omega_{0 . M I N}$ for the most unfavorable situation, is calculated by the formula

$$
\omega_{0 . M I N}=\sqrt{C_{G} / J_{0 . M A X}}
$$

where $J_{0 . M A X}$ - the maximum value of the moment of inertia of the manipulator mobile segment, driven by the drive under consideration. When the moment of inertia $J_{0}$ reduces, the frequency $\omega_{0}$ will increase, and the damping coefficient $\xi_{0}$ will increase too. Thus, the quality of the electromechanical subsystem is improved. In addition, the internal drive begins to exert an increasingly strong damping effect.

The cut-off frequency of the opened loop precision drive is determined by the formula

$$
\omega_{C} \leq \omega_{1} \xi_{2}
$$

from which it follows, that the cut-off frequency is higher than if there is no damping correction of velocity of the control object.

To increase the dynamic stiffness and accuracy of the drive at the time-varying impacts, it is advisable to increase the values of the frequencies $\omega_{0}$ and $\omega_{C}$. An increase of the stiffness coefficient and of the gear ratio also effects the accuracy positively.

\section{The results of the computer simulation of the high-precision geared servo drive with two motors}

A research of the dynamic properties of the proposed type of the precision servo drive with two motors is carried out on the example of the rotary drive of the robot manipulator with an open kinematic chain of the type PUMA in relation to the vertical axis. The static torque is absent. It is necessary to ensure that the proposed solution has the high quality of the control at the absence of intensive self-oscillations in the system, including an elastic gear with backlash in its position control loop of the massive control object.

The properties of servo motor KEB B3 and precision Harmonic Drive gear HDUC-2A are reflected in the mathematical model and the simulation program of the drive based on the model. The values of the parameters of such motor can be found in [21]. Nominal speed of the motor is $4000 \mathrm{rpm}$, rated torque is $2 \mathrm{Nm}$, rotor inertia moment is $5.7 \cdot 10^{-5} \mathrm{kgm}^{2}$, motor EMF coefficient is $0.577 \mathrm{Vs}$, electromagnetic time constant is $3.2 \mu$ s and active resistance is 9.2 Ohms. Gear ratio of the gear of the size 50 is 100 , its stiffness coefficient is $250000 \mathrm{Nm} / \mathrm{rad}$ and backlash is 1 arc minute. The parameter $\chi_{G}$ is adopted $500 \mathrm{Nms} / \mathrm{rad}$. It is considered that the drive of the torque resistance generates a constant torque equal to $45 \mathrm{Nm}$. A precision encoder of the primary position feedback of the control object is selected to identify the potential accuracy of the drive. There will be a change on one unit of a decimal number, corresponding to the code at the quadrature counter output, when manipulator rotates at the angle $1.75 \cdot 10^{-6} \mathrm{rad}$. We suggest that the PWM frequency in the frequency converter of the drive is $20 \mathrm{kHz}$. The parameter values of the internal servo drive controllers are selected in accordance with the rules of adjustment of systems of subordinated control contours. Such rules are given, for example, in [20]. The encoder of the position feedback loop of the motor shaft has a resolution 5000 samples per revolution. The cut-off frequency of the opened position control loop of the internal servo drive equals to $105 \mathrm{rad} / \mathrm{s}$.

When the control object with an inertia moment equals to $10 \mathrm{kgm}^{2}$ is controlled only by the internal drive with closed position control loop of the motor shaft, the computer simulation of the control processes of the control object has revealed significant oscillations without a corrective velocity loop of the control object (Fig. 2). It is seen, that fluctuations of the rotation angle $a$ of the control object are weakly damped. They last more than one second when there is a step input impact of $0.01 \mathrm{rad}$. It is established that the resonant frequency of the electromechanical subsystem is approximately $35 \mathrm{rad} / \mathrm{s}$.

The obtained simulation result is the evidence of the fact that weakly damped electromechanical subsystem will not allow to increase accuracy of the servo drive significantly, as it constrains us to limit its cut-off frequency. 


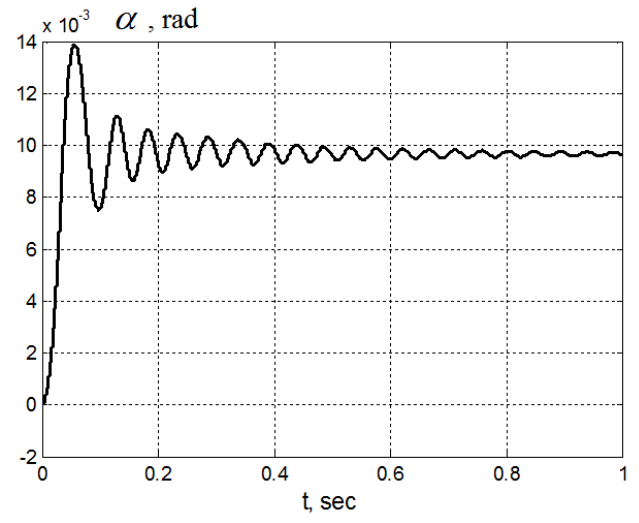

Fig. 2. The transient process of adjustment of the control object position by the internal drive without a corrective component

If we consider the closed loop precision servo drive with two motors, significant and slowly damped oscillations of the rotation angle $\alpha$ of the control object are also discovered when there is a reference impact of the rotation angle $\beta=0.25 \mathrm{rad}$ (Fig. 3). This simulation result is obtained at the absence of the corrective velocity feedback of the control object. It confirms the conclusion that, in spite of the high potential accuracy of the drive under consideration, its implementation for technological robots is impossible without introducing a corrective link, effecting the impedance.

The introduction of the corrective velocity feedback of the control object has the required damping effect and fundamentally changes the nature of the object movement. At a feedback coefficient $k_{V F}=9000 \mathrm{~s}$ the transient process acquires the form shown in Fig. 4. It can be seen that the oscillations caused by the elasticity of the gear are fully damped. The adjustment of the the controllers provides the values of the cut-off frequencies of the opened loop precision drive $\omega_{C}$ and of the opened loop internal drive $\omega_{C 2}$ as $8.97 \mathrm{~s}^{-1}$ and $127 \mathrm{~s}^{-1}$, respectively.

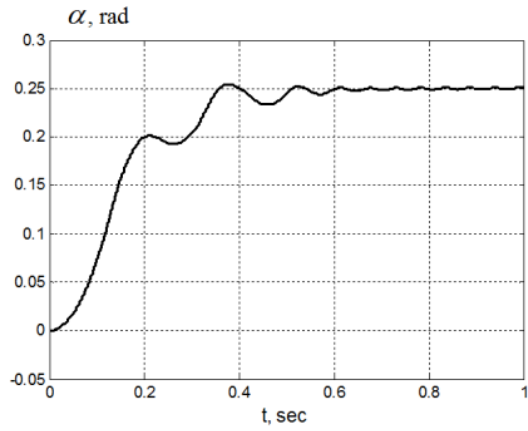

Fig. 3. The transient process of adjustment of the control object position by the precision drive without a corrective velocity feedback of the control object

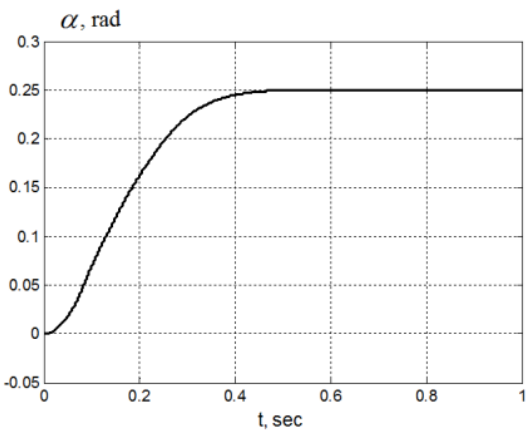

Fig. 4. The transient process of adjustment of the control object position by the precision drive with a corrective velocity feedback of the control object

Computer researches have confirmed the high accuracy of the servo drive with two motors under consideration. The drive error practically aspires to zero when there are constant reference impacts. After the completion of the transition process the mismatch of the drive $\delta=\beta-a$ makes minor fluctuations. At the torque $45 \mathrm{Nm}$ of the drive of the torque resistance the oscillation amplitude is $1.1 \cdot 10^{-7} \mathrm{rad}$. A negligible constant component of the error is observed, which approximately equals $-4 \cdot 10^{-8}$. Despite the fact that there are some oscillations, the elastic gear elements permanently remain tightened to each other, which provides high accuracy of the geared servo drive under consideration. These oscillations incidentally effect the positioning accuracy of the actuator, and so, their impact on the quality of technological process can be neglected.

When small initial misalignments are developed, for example, at $\beta=0.1 \mathrm{rad}$, at which the drive operates in the linear zone of its characteristics, the duration of the transient process equals to 0.25 seconds. This fact is the evidence of sufficiently high performance of the drive.

The variable reference impact $\beta$ is reproduced with high accuracy when the combined control is used. The simulation results have confirmed the theoretical conclusion of the necessity of a velocity feedback of variation of the reference impact with a coefficient $k_{\text {comp }}=\omega_{C}^{-1}=0.1114 \mathrm{~s}$. With such control, the error amplitude of the drive does not exceed $1.9 \cdot 10^{-4} \mathrm{rad}$ when the reference impact is developed. In this case, the reference impact varies with the amplitude $0.1 \mathrm{rad}$ and angular frequency $1 \mathrm{rad} / \mathrm{s}$. At a program control, the rate of the reference impact variation can be calculated 
at the analytical relations, in particular, as proposed in [22].

It is established during the simulation process that the limitations proper to the drive elements have a considerable influence on the drive when there is a significant initial mismatch. For example, when a step reference impact is $\beta=5$ rad, the primary time of the transient process passes at the maximum possible speed of $2 \mathrm{rad} / \mathrm{s}$ (Fig.5). Therefore, to ensure stability of control processes at large initial mismatches and to eliminate significant overcontrol it is advisable to implement non-linear correction of the impact, supplied to the input of the position controller of the precision drive, described, for example in [20].

The simulation results are also the evidence of high stability of the properties of the servo drive. They have shown that when the inertia moment of the control object reduces from 10 to $1 \mathrm{kgm}^{2}$, the high quality of the transient process remains at the same value of $k_{V F}$. At a more significant reduction of the inertia moment, it is advisable to carry out an adaptive adjustment of the value $k_{V F}$, in order to preserve stability margins of the corrective loop.

Computer research of the drive reaction to external force impact has shown that the constant external force impact is fully compensated in a steady-state regime, and the drive error $\delta$ becomes zero. At the harmonic variation of the moment of external forces the drive error, caused by it, also varies harmonically in the steady-state regime. When the amplitude of the external moment is $M_{V A R}=1 \mathrm{Nm}$, the maximum error amplitude, equal to $4 \cdot 10^{-6} \mathrm{rad}$, is observed at the angular frequency of oscillations of the moment of external forces $\omega_{M}=20 \mathrm{rad} / \mathrm{s}$ (Fig. 6).

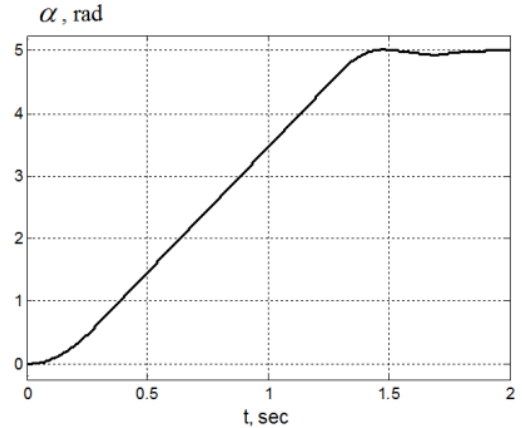

Fig. 5. Testing a large starting mismatch by a precision drive with a corrective velocity feedback of the control object and a nonlinear correction of the input impact of the position controller

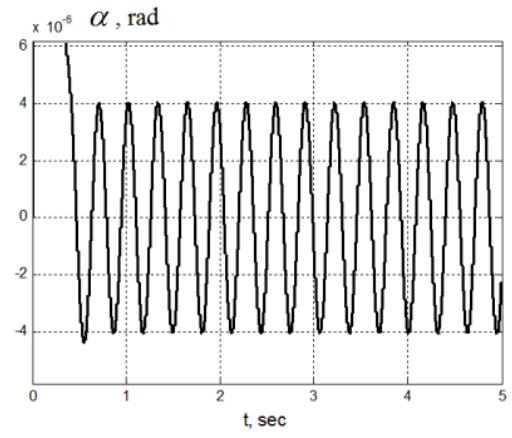

Fig. 6. Variation of the servo drive error at harmonic variation of the moment of external forces with an amplitude of $1 \mathrm{Nm}$ and a frequency of $20 \mathrm{rad} / \mathrm{s}$

It is deduced as a result of the simulations, that the position error of the control object is directly proportional to the amplitude of the oscillations of the moment of the external forces $M_{V A R}$ and frequency $\omega_{M}$, and when it is less than 20 $\mathrm{rad} / \mathrm{s}$. In particular, at the amplitude $M_{V A R}=1 \mathrm{Nm}$ and $\omega_{M}=1 \mathrm{rad} / \mathrm{s}$, the amplitude of the error $\delta$ equals to $4.4 \cdot 10^{-7}$ rad. When the angular frequency $\omega_{M}$ is higher than $20 \mathrm{rad} / \mathrm{s}$, there will be a decrease of the amplitude $\delta$ too, due to the damping action of the massive control object. In particular, at $\omega_{M}=500 \mathrm{rad} / \mathrm{s}$ the amplitude $\delta$ does not exceed $5 \cdot 10^{-7}$ $\mathrm{rad}$. The error of the effector position, caused by the dynamic stiffness of the drive, does not exceed 4 micrometres in the worst case, when the effector is at a distance of $1 \mathrm{~m}$ from the rotation axis of the output shaft of the drive and when $\omega_{M}=20 \mathrm{rad} / \mathrm{s}$. This error can be neglected in the most cases, proper to the technological robotics.

The results of the theoretical analysis and the computer simulation have answered the central issues of the research. They are the evidence of the fact that the recommended structure of the servo drive with impedance control allows to achieve a significant increase of the dynamic stiffness and oscillation damping of the control object. It can be assumed that the drive stiffness tends to infinity at the constant external moment, and at the varying moment of external forces, the stiffness is large enough to solve many practically significant problems. Thus, the geared drive with two motors under consideration can be attributed to the class of high-precision servo drives and it can be recommended for the construction of technological robots.

\section{Conclusion}

The research results have shown the advisability of application of precision geared servo drives with two motors in technological robots. The central task for the creation of such servo drives is concluded in exclusion of the limit cycles and in application of the controlled damping of oscillation of electromechanical subsystems.

To solve this problem the structure of servo drive is suggested. Its feature is an existence of four control contours. One of them serves for the position control of the manipulator link. The second and the third ones are used to control the position of the shaft of the primary motor and the torque, developed by the second motor - the drive of the torque resistance, respectively. And the fourth contour is designed to correct the dynamic properties of the drive in order to damp weakly damped oscillations of the control object. An idea of the impedance control is realized at that. The obtained results convince us that such servo drive has high stiffness and sufficient damping. 
It is possible to achieve high quality of the geared servo drive only when we introduce corrective links of the rotation velocity of the manipulator links. It expands the bandwidth of the drives and increases their performance and dynamic stiffness. The drive stiffness goes to infinity at a stall position of the control object. Positioning accuracy depends only on the error and the resolution of the sensor, which measures directly the position of the control object. It is especially important at analytical programming of technological robots.

Thus, the considered geared servo drive with two motors with the suggested variant of impedance control can be referred to a class of precision drives with significant dynamic stiffness and high damping. Such drive can be recommended for the construction of technological robots. The given mathematical relations form the basis for the choice of the values of its parameters.

The obtained results give a possibility to focus on reducing other components of the positioning error of the effector of technological robot, for example, caused by imperfection of the mathematical model, used to control it. Moreover, an adaptive adjustment of the transfer coefficients of the corrective links may be required, taking into account the properties of the manipulation mechanism varying in a considerable range in course of technological operation.

\section{References}

[1] V. V. Bushuev, A. G. Ostretsov. Designing of gear hobbers of new generation on the basis of mechatronic modules, in: MSTU Stankin herald, volume №3 (26), MSTU Stankin, Moscow, 2013.

[2] Yu.V. Ilyukhin, Yu.V. Poduraev, Design of the executive systems of robots. Linearized systems, MPI, Moscow, 1989.

[3] E.S. Blaise, A. Zimin, E.S. Ivanov, Yu.V. Ilyukhin, Yu.N. Semenov, B.K. Chemodanov et al., Servo drives, T.1.: theory and design of servo drives, B.K. Chemodanov, BMSTU, Moscow, 1999.

[4] M. Slamani, I. Bonev, Characterization and experimental evaluation of gear transmission errors in an industrial robot, in: Industrial Robot: An International Journal, volume 40, issue 5 (2013), pp. 441-449.

[5] Yu.A. Bortsov, G.G. Sokolovsky, Automated electric drive with elastic ties, Energoatomizdat, Saint Petersburg, 1992.

[6] V. A. Kuzovkin, V. V. Filatov, M. V. Chumaeva, Nonlinear models of dynamic processes in impulse control systems of brushless DC motors, in: Journal of computer and systems sciences international, volume 53, issue 3, May 2014, pp. 420-429.

[7] A. G. Andreev, M. M. Stebulyanin, Formation of stator currents in the simulation of switched electric drives in robots, in: Russian Engineering Research, volume 30, issue 10, October 2010, pp 1046-1052.

[8] T. Wescott, Applied Control Theory for Embedded Systems, Elsevier, 2006, pp. 320.

[9] X. Shen, J. HU, M. Zhang, L. Zhang, Experimental study on backlash compensation of CNC machine tool, in: Advanced Materials Research, volume 580, Trans tech Publications, Switzerland, 2012, pp. 419-422.

[10] B. Feng, X.S. Mei, L. Guo, D.S. Zhang, Y.L. Cheng, Backlash compensation on CNC machine tool based on semiclosed loop control, in: Advanced Materials Research, volume 346, September 2011, pp. 644-649.

[11] S.G. Robertz, L. Halt, S. Kelkar, K. Nilsson, A. Robertsson, D. Schar, J. Schiffer, Precise robot motions using dual motor control, in: IEEE International Conference on Robotics and Automation, Anchorage, 2010, pp. 5613 - 5620.

[12] M. Švaco, B. Šekoranja, F. Šuligoj, B. Jerbic, Calibration of an Industrial Robot using a Stereo Vision System // 24th DAAAM International Symposium on Intelligent Manufacturing and Automation, DAAAM 2013. Procedia Engineering 69 (2014), Volume 100, pp. 459 - 463.

[13] Albert Nubiola, Ilian A. Bonev, Geometric approach to solving the inverse displacement problem of calibrated decoupled 6R serial robots. Transactions of the Canadian Society for Mechanical Engineering, 2014, Vol. 38, №1, pp. 31-44.

[14] SINUMERIK 840D sl, 828D, Special functions, Function Manual, Nurnberg, Siemens, 2012, pp. 499 - 530.

[15] J. Schiffer, Dual motor control for backlash reduction, Master Thesis, Lund University Department of Automatic Control, Lund, Sweden 2009.

[16] M Mosadeghzad, GA Medrano-Cerda, JA Saglia, NG Tsagarakis, and DG Caldwell, "Comparison of Various Active Impedance Control Approaches, Modeling, Implementation, Passivity, Stability and Trade-offs," in IEEE/ASME International Conference on Advanced Intelligent Mechatronics, pp. 342-348, July 2012

[17] M Mosadeghzad, GA Medrano-Cerda, JA Saglia, NG Tsagarakis, and DG Caldwell, "Impedance control of a class of series elastic actuators: performance limitations arising from link dynamics, disturbance attenuation and impedance emulation," in IEEE Int. Conf. on Robotics and Biomimetics (ROBIO), Dec 2014

[18] Y Zhao, N Paine, and L Sentis, "Sensitivity comparison to loop latencies between damping versus stiffness feedback control action in distributed controllers," in ASME 2014 Dynamic Systems and Control Conference, DSCC2014, October 2014

[19] C Chen, H Nie, J Chen, and X Wang, “A velocity-based impedance control system for a low impact docking mechanism (LIDM)," in Sensors 14(12):22998-23016, December 2014

[20] Yu.V. Ilyukhin, Computer control of mechatronic systems, MSTU "STANKIN", Moscow, 2014.

[21] KEB Servo Motors, KEB instruction manual, Barntrup, June 2004.

[22] Yu.V. Ilyukhin, Yu.V. Poduraev, A.V. Tatarintseva. Nonlinear Adaptive Correction of Continuous Path Speed of the Tool for High Efficiency Robotic Machining // 25th DAAAM International Symposium on Intelligent Manufacturing and Automation, DAAAM 2014. Procedia Engineering (2015), Volume 100, pp. 994-1002. 